\title{
PRODUTIVIDADE E QUALIDADE DA UVA 'CABERNET SAUVIGNON' PRODUZIDA SOB COBERTURA DE PLÁSTICO EM CULTIVO ORGÂNICO’
}

\author{
ALESSANDRA MARIA DETONI², EDMAR CLEMENTE ${ }^{3}$, CARLINHOS FORNARI ${ }^{4}$
}

RESUMO-A uva 'Cabernet Sauvignon' (Vitis vinifera L.) é utilizada na produção de vinhos finos, sendo muito cultivada no Sul do Brasil. Esta variedade é muito sensível à ocorrência de doenças, sendo necessário o desenvolvimento de práticas culturais para diminuir a incidência das mesmas. O objetivo deste trabalho foi avaliar a produtividade e a qualidade da uva 'Cabernet Sauvignon' cultivada sob cobertura de plástico em sistema de produção orgânico. O experimento foi realizado em um vinhedo localizado no município de Toledo, região oeste do Paraná, sendo as plantas conduzidas no sistema de espaldeira, com cobertura de plástico na linha de plantio. Foram determinados: teor de sólidos solúveis (SS), a acidez titulável (AT), pH, antocianinas totais, produtividade, número de cachos por planta e peso médio dos cachos. Não foram encontradas diferenças significativas no teor de SS (17,3 ${ }^{\circ}$ Brix), porém os frutos sob a cobertura plástica apresentaram maiores teores de AT e $\mathrm{pH}, 1,14 \mathrm{~g} 100 \mathrm{~mL}^{-1}$ de suco e 3,4, respectivamente, que aqueles

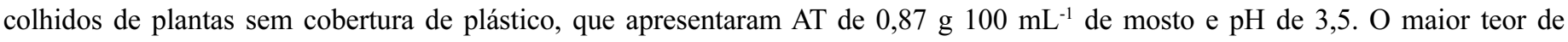
antocianinas totais foi verificado nas plantas fora da cobertura, com 22,8 $\mathrm{mg} \mathrm{L}^{-1}$. Nas plantas protegidas, a produção foi maior (1769 $\mathrm{g}$ planta $\left.^{-1}\right)$ do que nas plantas sem cobertura $\left(492 \mathrm{~g} \mathrm{planta}^{-1}\right)$, que apresentaram elevado índice de doenças. Conclui-se, desta forma, que a cobertura de plástico viabiliza o cultivo da uva 'Cabernet Sauvignon' no sistema de produção orgânico, por proporcionar diminuição na incidência de doenças.

Termos para indexação: viticultura, ambiente protegido, Vitis vinifera, enologia.

\section{PRODUCTIVITY AND QUALITY OF GRAPE ‘CABERNET SAUVIGNON’ PRODUCED IN ORGANIC SISTEM UNDER PLASTIC COVERING}

\begin{abstract}
The grape 'Cabernet Sauvignon' (Vitis vinifera L.) it is used in fine wines production, being very cultivated in the South of Brazil. This variety is very sensitive of occurrence of diseases, being necessary the development of cultural practices to reduce the incidence of the same ones. The objective of this work was to evaluate the productivity and the quality of the grape 'Cabernet Sauvignon' cultivated under covering of plastic in organic production system. The experiment was accomplished in an organic orchard in the west area of the state of Paraná; the plants were conducted in espalier system, with plastic covering in the planting line. It was evaluated tenor of soluble solids (SS), titrable acidity (TA), $\mathrm{pH}$, total antocyanins, productivity, number of bunches for plant and medium bunch weight. They were not found significant differences in the tenor of SS (17.3 $3^{\circ}$ rix), however the fruits under the covering

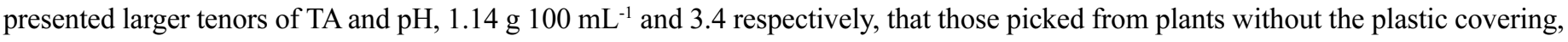

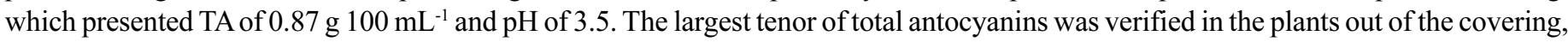
with $22.8 \mathrm{mg} \mathrm{L}^{-1}$. In the protected plants, the production was larger $\left(1769 \mathrm{~g} \mathrm{plant}^{-1}\right)$ than in the plants without covering $\left(492 \mathrm{~g} \mathrm{plant}^{-1}\right)$, which presented high index of diseases. It is concluded that the plastic covering makes possible the cultivation of grape 'Cabernet Sauvignon' in the organic production system, for providing a decrease in the incidence of diseases.
\end{abstract}

Index Terms: Viticulture, plastic greenhouse, Vitis vinifera, enology.

\section{INTRODUÇÃO}

A videira 'Cabernet Sauvignon' (Vitis vinifera L.) é originária de Bordeaux, França, sendo uma das uvas viníferas mais cultivadas no Brasil (Giovannini, 2001; Pommer et al., 2003). De sabor herbáceo, destina-se à elaboração de vinho tinto de guarda, o qual requer amadurecimento e envelhecimento, ou de vinho para ser consumido jovem (Rizzon \& Miele, 2002). Apresenta os cachos cilíndricos e longos, pesando em média 130 a 170 g, sendo as bagas pequenas, esféricas e pretas (Pommer et al., 2003). Sua produtividade varia de 15 a $20 \mathrm{t} \mathrm{ha}^{-1}$, com teores de açúcar entre 16 e $18^{\circ}$ Brix (Giovaninni, 2001).

As principais regiões vitícolas do Sul e Sudeste do Brasil são caracterizadas por apresentarem umidade e temperaturas elevadas, aliadas a precipitações freqüentes durante o ciclo vegetativo da videira (Sônego et al., 2005). Essas condições impõem maiores dificuldades no cultivo, principalmente no que se refere à maior ocorrência de doenças fúngicas da parte aérea $\mathrm{e}$ no controle das mesmas. A viabilização da viticultura nestas

1(Trabalho 202-06). Recebido em: 30-11-2006. Aceito para publicação em: 24-08-2007.

2Doutoranda em Agronomia, UEM- Av.Colombo,5790- CEP 87020-900. Maringá-PR. e-mail: alessandradetoni@yahoo.com.br.

${ }^{3}$ Químico,PhD em Ciência dos Alimentos - UEM. Av.Colombo,5790- CEP 87020-900 .Maringá-PR.e-mail: eclemente@uem.br.

${ }^{4}$ Engenheiro Químico, Fundação para o Desenvolvimento Científico e Tecnológico de Toledo - FUNTEC, Toledo, Paraná. E-mail: fornari@toledonet.com.br. 
condições, para a maioria das variedades cultivadas, requer a aplicação preventiva e freqüente de produtos químicos para o controle das doenças fúngicas da parte aérea durante o ciclo vegetativo.

Para Giovannini (2001), as uvas viníferas finas são as mais sensíveis às doenças, sendo difícil seu controle em sistemas agroecológicos. Com o uso, porém, de cobertura de plástico, espera-se diminuir o molhamento foliar e, com isso, diminuir a incidência de doenças e obter êxito no cultivo de uvas viníferas no sistema de produção orgânico.

$\mathrm{O}$ uso de cobertura de plástico tem sido uma das alternativas mais usadas por viticultores de outros países para antecipar ou retardar a colheita e também para controlar condições ambientais adversas (Fao, 1990; Gattorta et al., 1976; Manzo \& Ruggieri, 1988; Olivelli, 1976). No Brasil, essa técnica já foi testada com sucesso no Rio Grande do Sul (Schiedeck, 1996). As principais vantagens verificadas com o uso foram a redução dos tratamentos fitossanitários, melhor qualidade dos frutos, economia de insumos e conservação do solo, tudo isso associado à valorização comercial do produto pela obtenção de colheitas fora de época.

A redução dos tratamentos fitossanitários com o uso da cobertura de plástico torna-se uma oportunidade, viabilizando a viticultura orgânica, a qual dispensa o uso de agrotóxicos e fertilizantes, que causam desequilíbrios ou que sejam agressivos à saúde e ao meio ambiente (Penteado, 2000). A exigência do certificado ambiental é uma tendência mundial e tornou-se um excelente instrumento de mudança de comportamento dos viticultores, criando para eles uma nova alternativa de mercado (Choudhury et al., 2001).

Por outro lado, frutos protegidos com cobertura de plástico não correm riscos ambientais durante a maturação, o que permite planejar a colheita na época mais conveniente e obter matériaprima de melhor qualidade para a produção do vinho (Avelant \& Loubser, 1998). Diante disso, o objetivo deste trabalho foi avaliar a produtividade e a qualidade da uva 'Cabernet Sauvignon' sob cobertura de plástico em cultivo orgânico.

\section{MATERIAL E MÉTODOS}

O trabalho foi realizado em vinhedo comercial de uvas 'Cabernet Sauvignon', enxertadas sobre o porta-enxerto 420 - A, na cidade de Toledo - PR, na safra 2005-2006. As plantas foram conduzidas no sistema de espaldeira, com espaçamento de $1,5 \mathrm{~m}$ entre plantas na linha e 3,0 m nas entrelinhas. O pomar foi organizado de acordo com o sistema de produção orgânico, com certificado de produtor orgânico expedido pelo IBD (Instituto Biodinâmico de Desenvolvimento Rural). Portanto, todas as práticas culturais realizadas no pomar foram efetuadas de acordo com o que é permitido pela entidade certificadora.

O delineamento experimental utilizado foi o inteiramente casualizado, onde cada tratamento (1 - Plantas cobertas, e 2 Plantas sem cobertura) possuía 10 repetições constituídas por 3 plantas, totalizando 30 plantas para cada tratamento, além das plantas pertencentes à bordadura, as quais não foram avaliadas. A cobertura de plástico foi feita por meio da utilização de tecido plano de polietileno (PROPEX Fabrics ${ }^{\grave{O}}$ ), com 170 micras de espessura, de coloração natural, e com $80 \%$ de passagem de luz. Para a sustentação do tecido plástico, foi construída uma estrutura de madeira, apoiada nos mourões de sustentação de cada linha da espaldeira, interligada com fios de arame, no sentido longitudinal, para a fixação da lona, conforme a Figura 1. Os arames foram envoltos com mangueira plástica, para evitar danos de atrito ao tecido. Entre cada estrutura de cobertura, ficou um espaço de aproximadamente $25 \mathrm{~cm}$, para que a água da chuva pudesse atingir apenas o sistema radicular das plantas, sem molhar o dossel vegetativo. A verificação do molhamento foliar, devido ao orvalho e às chuvas, foi feita visualmente, através da observação visual das plantas pela manhã.

Como foi constatada maior ocorrência de antracnose (Elsinoe ampelina), utilizaram-se, para a avaliação da incidência dessa doença, os mesmos critérios adotados por Pereira (2001). Nessa avaliação, que foi realizada quinzenalmente, são atribuídas as notas $1 ; 3 ; 5 ; 7 \mathrm{e} 9$, referentes aos níveis de ataque de antracnose, os quais evoluem de ausente, para leve, médio, forte e muito forte, respectivamente.

Para a colheita dos cachos, efetuou-se uma amostragem de bagas para avaliar o teor de sólidos solúveis totais, expresso em ${ }^{\circ}$ Brix, através de leitura em refratômetro manual. Os cachos foram colhidos manualmente, quando apresentaram, no mínimo, $17^{\circ}$ Brix, com auxílio de tesoura de poda, sendo posteriormente submetidos à limpeza, retirando-se folhas ou ramos contidos entre as bagas de uvas, bagas danificadas, podres ou picadas por insetos.

A produtividade foi determinada por meio da pesagem de toda a uva colhida, com auxílio de balança semi-analítica, e as avaliações químicas foram feitas no mosto, obtido após o desengace e esmagamento das bagas. Para as avaliações químicas, foram feitas as seguintes determinações: teor de sólidos solúveis (SS), obtido por refratometria (AOAC, 1992); acidez titulável (AT), com metodologia descrita por Carvalho et al. (1990); pH por meio de pHmetro digital (AOAC, 1992), e antocianinas totais por espectrofotometria, com leitura a $520 \mathrm{~nm}$, segundo Bevilaqua (1995). Os dados foram analisados por meio do programa estatístico SISVAR, versão 4.3 (Ferreira, 2000).

\section{RESULTADOS E DISCUSSÃO}

Na Tabela 1, estão os resultados obtidos em relação às características químicas do mosto. Nessa tabela, observa-se que, em relação ao teor de sólidos solúveis, o valor médio em ambos os tratamentos foi de $17,3^{\circ}$ Brix. Rizzon \& Miele (2002), ao avaliarem algumas características químicas do mosto da uva 'Cabernet Sauvignon' cultivada em Bento Gonçalves-RS, no período de 1987 e 1992, observaram, em média, $18,1^{\circ}$ Brix.

Ferreira et al. (2004) não observaram diferenças estatísticas em relação ao teor de sólidos solúveis da uva 'Cabernet Sauvignon' quando cultivada sob cobertura de plástico, em Jundiaí-SP, onde o teor de sólidos solúveis variou de 13,7 a $14,7^{\circ}$ Brix nas plantas sob a cobertura e de 13,6 a $15,3^{\circ}$ Brix nas plantas cultivadas a céu aberto. Esses últimos autores sugerem que o nível de modificação alcançado com a introdução da 
cobertura de plástico no vinhedo, por si só, não foi suficiente para modificar esse ambiente com possibilidade de incrementar o acúmulo do teor de sólidos solúveis, o que também foi verificado no presente trabalho.

Analisando a Tabela 1, verificam-se diferenças significativas entre os tratamentos em relação ao $\mathrm{pH}$ e à acidez titulável (AT). As plantas sob a cobertura de plástico apresentaram maior acidez, com um teor de acidez titulável de $1,14 \mathrm{~g}$ de ácido tartárico em $100 \mathrm{~mL}$ de suco e $\mathrm{pH}$ de 3,4 ; já nas plantas sem

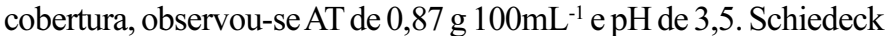
(1996) também relata maiores valores de acidez total do mosto da uva quando a planta é cultivada sob cobertura de plástico.

Em relação ao teor de antocianinas (Tabela 1), observa-se que as uvas fora da cobertura de plástico apresentaram maior teor de antocianinas, em média de $22,8 \mathrm{mg} \mathrm{L}^{-1}$, em relação às plantas sob cobertura de plástico, $14,9 \mathrm{mg} \mathrm{L}^{-1}$. Apesar de terem sido detectadas menores quantidades de antocianinas nas uvas sob a cobertura de plástico, não se observou diferença no aspecto visual de sua coloração. Manzo \& Ruggieri (1988) verificaram que a uva cv. Matilde, cultivada em estufa, apresentou bagas com coloração menos intensa do que as cultivadas a céu aberto, porém foram aceitas normalmente pelos consumidores, não causando prejuízos na comercialização.

Na Tabela 2, referente aos dados de produção, observa-se uma baixa produtividade nas plantas avaliadas, independentemente do uso ou não da cobertura de plástico. A produtividade média dos tratamentos, $3,0 \mathrm{tha}^{-1}$, está bem abaixo da média observada para a cultivar 'Cabernet Sauvignon', que gira em torno de 15 a 20 tha $^{-1}$ (Giovannini, 2001).

Porém, apesar da baixa produtividade observada no presente estudo, as plantas cultivadas sob a cobertura de plástico apresentaram valores superiores $\left(4,0 \mathrm{tha}^{-1}\right)$ em relação às plantas sem cobertura $\left(1,0 \mathrm{t} \mathrm{ha}^{-1}\right)$. Essa queda de produção pode ser justificada, em parte, pelo fato de que, durante a maturação dos cachos, ocorreu um intenso ataque de abelhas, as quais causaram muitos danos nas bagas, diminuindo o peso dos cachos e dificultando o processo de colheita. De acordo com os dados da Tabela 2, os cachos pesaram, em média, $84 \mathrm{~g}$ nas plantas cobertas e $49 \mathrm{~g}$ nas plantas sem cobertura. Esses dados são inferiores ao relatado por Pommer et al. (2003), que citam que os cachos de 'Cabernet Sauvignon' pesam, em média, de 130 a 170 g. Já Rizzon $\&$ Miele (2002) reportam que os cachos de 'Cabernet Sauvignon', cultivada em Bento Gonçalves, apresentaram peso médio de $149,3 \mathrm{~g}$.

Em relação à produção por planta (Tabela 2), observou-se, em média, $1.769 \mathrm{~g} \mathrm{planta}^{-1}$ cultivada sob a cobertura de plástico, superior àquelas fora da cobertura, que apresentaram, em média, 492 g planta $^{-1}$. Ferreira et al. (2004) verificaram maiores valores de produção nas plantas de 'Cabernet Sauvignon', sob diferentes porta-enxertos, cultivadas sem o uso de cobertura de plástico (638 a $1.507 \mathrm{~g} \mathrm{planta}^{-1}$ ), do que em plantas com o uso da cobertura (606 a 1.400 g planta $^{-1}$ ).

Ao avaliar os dados da Tabela 3 , referentes à presença de antracnose nas plantas e frutos, pode-se observar que, no tratamento com cobertura plástica, apenas $33,3 \%$ das plantas foram afetadas, com nível 3 de doença. Na escala utilizada para a avaliação da incidência de antracnose, o número 3 indica ataque leve, com a presença de lesões pequenas apenas nas folhas, o que não trouxe grandes danos às plantas. Já nas plantas cultivadas a céu aberto, $80 \%$ das plantas obtiveram classificação 7 , que indica ataque forte, com lesões grandes, presentes nas folhas, ramos e cachos, demonstrando que elas foram muito prejudicadas com a presença da antracnose.

A umidade relativa do ar e a duração do período de molhamento foliar por orvalho estão intimamente ligadas, sendo que as principais doenças fúngicas ocorrem em condições de elevada umidade relativa e na presença de um filme de água sobre as folhas e frutos, de modo a propiciar a instalação do patógeno (Sentelhas, 1998). Nas plantas sob a cobertura, não foi observado, visualmente, o molhamento foliar nas primeiras horas do dia, em função do orvalho, nem após períodos chuvosos. O orvalho formado concentrava-se na região inferior da lona, sem deposição foliar. Além disso, a cobertura de plástico também protegeu as folhas do molhamento pela chuva, a qual atingiu apenas o sistema radicular das plantas, devido à abertura existente entre as estruturas individuais de madeira, instaladas em cada linha de cultivo, e que sustentavam as coberturas plásticas.

Já nas plantas sem cobertura, foi verificado o molhamento foliar através do orvalho e das chuvas, as quais foram muito freqüentes, da brotação até a colheita dos frutos, favorecendo, dessa forma, a incidência de antracnose nas plantas localizadas fora da cobertura de plástico. Schiedeck (1996) também relata que, no período de maturação, o ataque de pássaros e as condições meterológica, como chuvas e ventos, comprometeram consideravelmente a qualidade visual dos cachos das plantas cultivadas a céu aberto. No presente estudo, além de prejudicar a qualidade visual dos cachos, a maior ocorrência de antracnose nas plantas a céu aberto também provocou prejuízos na produção, com menor peso de cacho, número de cachos por planta e produção por área, conforme consta na Tabela 2.

Em relação a época de colheita dos frutos, não foram observadas diferenças entre os tratamentos, devido ao amadurecimento dos cachos ter ocorrido simultaneamente, diferentemente do que relata Schiedeck (1996), que, ao avaliar a variedade Niágara rosada, observou antecipação da maturação nas plantas cultivadas sob estufa de plástico.

TABELA 1 - Dados médios de sólidos solúveis (SS) em ${ }^{\circ}$ Brix, $\mathrm{pH}$, acidez titulável (AT) g de ácido tartárico em $100 \mathrm{~mL}$ e antocianinas totais em $\mathrm{mg} \mathrm{L}^{-1}$ de mosto de uvas 'Cabernet Sauvignon' produzidas em sistema de cultivo orgânico com e sem o uso de cobertura de plástico. Toledo-PR, 2006.

\begin{tabular}{lcccc}
\hline Tratamentos & SS & AT & pH & $\begin{array}{c}\text { Antocianinas } \\
\text { totais }\end{array}$ \\
\hline Cobertura & $17,3 \mathrm{a}^{*}$ & $1,14 \mathrm{a}$ & $3,4 \mathrm{~b}$ & $14,9 \mathrm{~b}$ \\
Sem cobertura & $17,3 \mathrm{a}$ & $0,87 \mathrm{~b}$ & $3,5 \mathrm{a}$ & $22,8 \mathrm{a}$ \\
\hline $\mathrm{CV}(\%)$ & 4,08 & 0,43 & 0,21 & 5,71 \\
\hline
\end{tabular}

* Médias seguidas de letras distintas na coluna diferem entre si, pelo teste

$\mathrm{F}$, ao nível de $5 \%$ de probabilidade. 
TABELA 2 - Médias de número de cachos planta $^{-1}$, peso de cacho $(\mathrm{g})$, produção planta ${ }^{-1}(\mathrm{~g})$ e produtividade $\mathrm{t}$ $\mathrm{ha}^{-1}$ de uvas 'Cabernet Sauvignon' produzidas em sistema de cultivo orgânico com e sem o uso de cobertura de plástico. Toledo-PR, 2006.

\begin{tabular}{lccccc}
\hline Tratamentos & $\begin{array}{c}\mathbf{N}^{\mathbf{0}} \text { cachos } \\
\text { planta }^{-1}\end{array}$ & $\begin{array}{c}\text { Peso de } \\
\text { cacho (g) }\end{array}$ & $\begin{array}{c}\text { Produção } \\
\text { planta }^{-1} \mathbf{( g )}\end{array}$ & $\begin{array}{c}\text { Produtividade } \\
\text { t ha }^{-1}\end{array}$ \\
\hline Cobertura & $21 \mathrm{a}^{*}$ & $84 \mathrm{a}$ & $1769 \mathrm{a}$ & $4,0 \mathrm{a}$ \\
Sem cobertura & $10 \mathrm{~b}$ & $49 \mathrm{~b}$ & $492 \mathrm{~b}$ & $1,0 \mathrm{~b}$ \\
\hline CV (\%) & 24 & 16 & 8 & 12 \\
\hline
\end{tabular}

* Médias seguidas de letras distintas na coluna diferem entre si, pelo teste $\mathrm{F}$, ao nível de $5 \%$ de probabilidade.

TABELA 3 - Dados médios (\%) de plantas com sintomas do ataque de antracnose, em diferentes níveis de ataque, na videira 'Cabernet Sauvignon' cultivada em sistema orgânico com e sem o uso de cobertura de plástico. Toledo-PR, 2006.

\begin{tabular}{lccccc}
\hline Tratamentos & \multicolumn{5}{c}{ Níveis de antracnose } \\
\cline { 2 - 6 } & $\mathbf{1}$ & $\mathbf{3}$ & $\mathbf{5}$ & $\mathbf{7}$ & $\mathbf{9}$ \\
\cline { 2 - 6 } Com cobertura & $66,6 \%$ & $33,3 \%$ & $0 \%$ & $0 \%$ & $0 \%$ \\
Sem cobertura & $3,3 \%$ & $10 \%$ & $6,6 \%$ & $80 \%$ & $0 \%$ \\
\hline
\end{tabular}

*Escala de notas apresentada no item Material e Métodos.

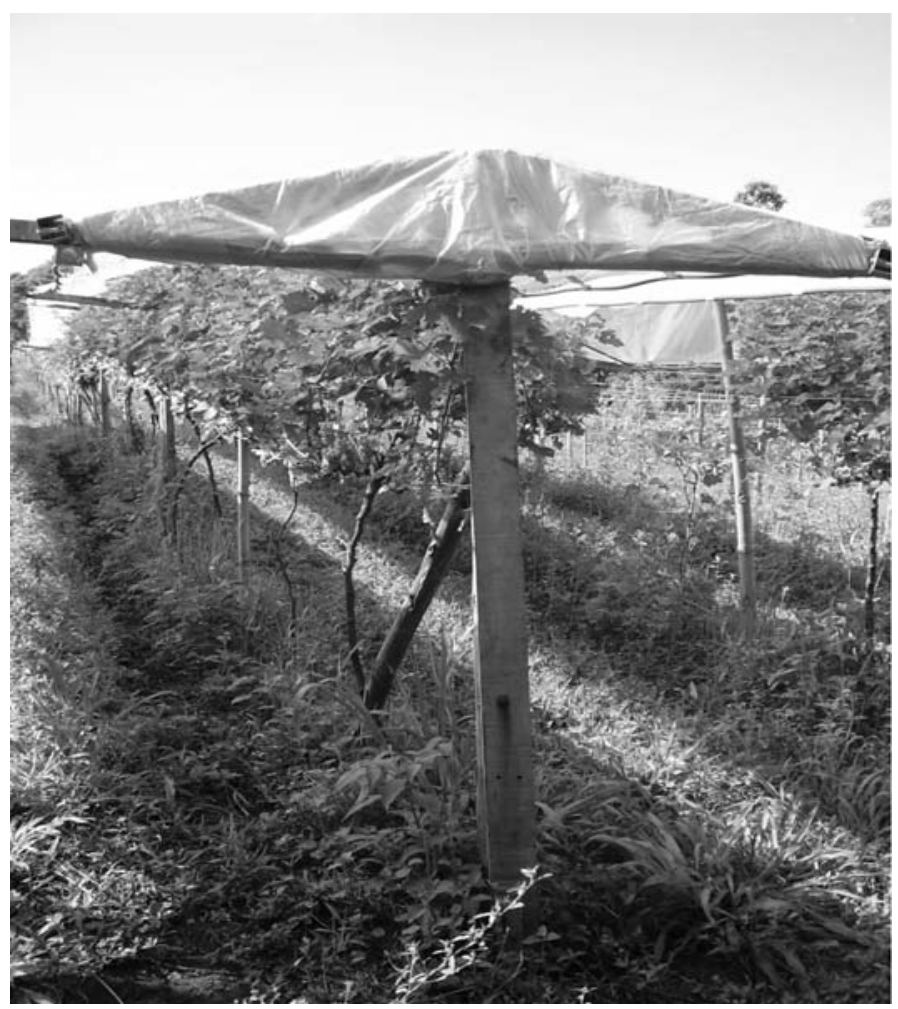

FIGURA 1 - Foto da estrutura de madeira utilizada para a fixação da cobertura de plástico nas linhas da espaldeira, em um pomar de uvas 'Cabernet Sauvignon', cultivada em sistema orgânico. Toledo PR-2006.

\section{CONCLUSÃO}

O uso da cobertura de plástico melhorou a qualidade física dos frutos e a produtividade, por ter diminuído a incidência de doenças nas plantas, porém não antecipou o período de colheita nem causou alterações químicas no mosto que pudessem prejudicar a qualidade química do material.

\section{REFERÊNCIAS}

AOAC - Association of Oficial Agriculture Chemists. Official methods of analysis of the association of the agricultural chemistry. $11^{\text {th }} \mathrm{ed}$. Washington, $1992.1115 \mathrm{p}$

AVELANT, J. H.; LOUBSER, J. T. The potential of overhead plastic covering for advanced ripening of table grapes. Deciduous Fruit Grower, Bellville, v.35, p.173-176, 1998.

BEVILAQUA, G. A. P. Avaliações físico-químicas durante a maturação de videiras cultivadas no Rio Grande do Sul. Revista Brasileira de Agrociência, Pelotas, v.1, n.3, p.151-156, 1995.

CARVALHO, C.R.L.; MANTOVANI, D.M.B.; CARVALHO, P.R.N.; MORAES, R.M.de. Análises químicas de alimentos. Campinas: Instituto de Tecnologia de Alimentos, 1990. 121p. Manual Técnico

CHOUDHURY, M.M.; COSTA, T.S. da; ARAÚJO, J.L.P. Agronegócio de uvas de mesa. In: CHOUDHURY, M.M (Coord.) Uva de mesa pós-colheita. Brasília: Embrapa Informação Tecnológica, 2001.p. 9-16. (Frutas do Brasil, 12).

FERREIRA, D. F. Manual do sistema Sisvar para análises estatísticas. Lavras: UFLA, 2000. 66 p.

FERREIRA, M. A.; PEDRO JÚNIOR, M. J.; SANTOS, A. O.; HERNANDES, J. L. Modificação parcial do ambiente de cultivo da videira 'Cabernert sauvignon' sobre diferentes porta-enxertos: efeito sobre a produção e o teor de sólidos solúveis totais. Bragantia, Campinas, v.63, n.3, p.439-445, 2004.

FOOD AGRICULTURE ORGANIZATION OF THE UNITED NATION. Plant production na protetion paper: protected cultivation in Mediterranean climat. Rome, 1990.313p.

GATTORTA, G.; AGNELLO, A.; LEANDRI,A.; IMBROGLINI, G. Influenza della copertura parziale del vigneto sulla entitá dei residui di fungicidi. Vignevini, Roma, v.3, n.2, p.11-14, 1976.

GIOVANNINI, E. Uva agroecológica. Porto Alegre: Renascença, 2001. 136p. 
MANZO, P.; RUGGIERI, L. M. Premiére expériences de semiforçage du raisin de table precoce "Matilde". Plasticulture, Paris, v.1, n.77, p.4-10, 1988

OLIVELLI, V. Protezione della vite dalla peronospora cin reti antigrandine in plástica impregnate di sali rameici. Vignevini, Roma, v. 3, n. 2, p. 23-27, 1976.

PENTEADO, S.R. Introdução à agricultura orgânica: normas e técnicas de cultivo. Campinas: Grafimagem, 2000.110 p.

PEREIRA, G. E. Caracterização agronômica de cultivares de videira para suco em Minas Gerais avaliação analítica e sensorial dos sucos. 2001. 126 f. Dissertação (Mestrado em Fitotecnia) Universidade Federal de Lavras, Lavras, 2001.

POMMER, C. V.; TERRA, M. M.; PIRES, E. J. P. Cultivares de videira. In: POMMER, C. V. Ed. Uva: tecnologia de produção, póscolheita, mercado. Porto Alegre: Cinco Continentes, 2003.p. 109-294.
RIZZON, L. A.; MIELE, A. Avaliação da cv. Cabernet sauvignon para elaboração de vinho tinto. Ciência e Tecnologia de Alimentos, Campinas, v. 22, n. 2, p. 192-198, 2002.

SCHIEDECK, G. Ecofisiologia da videira e qualidade da uva "Niágara rosada" conduzida sob estufa de plástico. 1996. $111 \mathrm{f}$ Dissertação (Mestrado em Fitotecnia) - Universidade Federal do Rio Grande do Sul, Porto Alegre, 1996.

SENTELHAS, P.C. Aspectos climáticos para a viticultura tropical. Informe Agropecuário, Belo Horizonte, v.19, n.194. p.9-14, 1998.

SÔNEGO, O. R.; GARRIDO, L. da R.; GRIGOLETTI JÚNIOR, A. Principais doenças fúngicas da videira no Sul do Brasil. Bento Gonçalves: Embrapa Uva e Vinho,2005. 32p. (Circular Técnica, 56). 